\title{
Tumor mutational burden is associated with poor outcomes in diffuse glioma
}

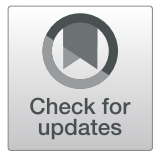

\author{
Lihong Wang ${ }^{1}$, Jia Ge', Yang Lan', Yu Shi', Ying Luo ${ }^{1}$, Yuhuan Tan ${ }^{1,2}$, Mei Liang ${ }^{1,2}$, Song Deng ${ }^{1,2}$, Xia Zhang ${ }^{1}$, \\ Wenying Wang ${ }^{1}$, Yaoyao Tan' ${ }^{1}$ Y Yuanyuan $\mathrm{Xu}^{1}$ and Tao Luo ${ }^{1 *}$
}

\begin{abstract}
Background: Tumor mutational burden (TMB) is a potential biomarker for immune checkpoint therapy and prognosis. The impact of TMB on clinical outcomes and the correlation coefficient between exome sequencing and targeted sequencing in glioma have not yet been explored.

Methods: Somatic mutations in the coding regions of 897 primary gliomas and the clinical and RNA-seq data of 654 patients in The Cancer Genome Atlas (TCGA) database were analyzed as a training set, while another 286 patients in the Chinese Glioma Genome Atlas (CGGA) database were used for validation. Descriptive and correlational analyses were conducted with TMB. Enrichment map analysis and gene set enrichment analysis (GSEA) were also performed.

Results: TMB was higher for the group of mutant genes that are frequently mutated in glioblastomas (GBMs) and lower for the group of mutant genes that are frequently mutated in lower-grade gliomas (LGGs). Patients with a higher TMB exhibited shorter overall survival. TMB was associated with grade, age, subtype and mutations affecting genomic structure. Moreover, univariate and multivariate analyses showed that TMB was an independent prognostic factor for glioma. The signaling pathways of the cell cycle were enriched in the TMB ${ }^{\text {High }}$ group. TMB was higher in the mismatch repair (MMR) gene mutant group than in the wild-type group, but the MMR pathway was enriched in the TMB ${ }^{\text {High }}$ group of gliomas without mutations in classical MMR genes. The correlation between TMBs calculated through exome sequencing and targeted sequencing was moderate, and panel-based TMB was not correlated with prognosis.

Conclusions: TMB is associated with poor outcomes in diffuse glioma. The high proliferative activity in the TMB High group could account for the shorter survival of these patients. This association was not reflected by a pan-cancer targeted sequencing panel.
\end{abstract}

Keywords: TMB, Glioma, Prognosis, Pan-cancer targeted sequencing

\section{Background}

Glioma is the most common malignant primary brain tumor in adults. Molecular classification via genomics, transcriptomics and methylomics has revealed the potential value of diagnosis based on molecules [1-3]. With the publication of the 2016 WHO classification,

\footnotetext{
* Correspondence: Ity3169@163.com

'Institute of Pathology and Southwest Cancer Center, Southwest Hospital, Third Military Medical University (Army Medical University) and Key Laboratory of Tumor Immunopathology, Ministry of Education of China, Chongqing 400038, China

Full list of author information is available at the end of the article
}

integrated diagnosis including mutational and histological phenotypes has been broadly applied in pathological typing. In some cases, the genotype even trumps the histological phenotype [4]. Tumor mutational burden (or tumor mutational load) is a potential biomarker of immune checkpoint inhibitors in many cancer types, as neoantigens are generated by somatic tumor mutations [5]. T cell-inflamed GEPs (gene expression profiles) are used to predict the response to PD-1 blockade and combined with TMB, they are used to predict the effects of anti-PD-1 treatment [6]. TMB is also a poor

C C The Author(s). 2020 Open Access This article is licensed under a Creative Commons Attribution 4.0 International License, which permits use, sharing, adaptation, distribution and reproduction in any medium or format, as long as you give appropriate credit to the original author(s) and the source, provide a link to the Creative Commons licence, and indicate if changes were made. The images or other third party material in this article are included in the article's Creative Commons licence, unless indicated otherwise in a credit line to the material. If material is not included in the article's Creative Commons licence and your intended use is not permitted by statutory regulation or exceeds the permitted use, you will need to obtain permission directly from the copyright holder. To view a copy of this licence, visit http://creativecommons.org/licenses/by/4.0/ The Creative Commons Public Domain Dedication waiver (http://creativecommons.org/publicdomain/zero/1.0/) applies to the data made available in this article, unless otherwise stated in a credit line to the data. 
prognostic marker for neuroblastoma but a good prognostic marker for non-small-cell lung cancer [7, 8]. Furthermore, deficiency of the MMR complex leads to the accumulation of mutations $[9,10]$. Exome sequencing or targeted sequencing is used to measure TMB. It is reported that panel-based TMB is highly correlated with TMB calculated by exome sequencing $[9,11]$; thus, panel-based TMB is commonly used in cancer patients to predict survival after immunotherapy [5].

In glioma, the reports of TMB seem to be controversial. TMB is higher in LGG than in GBM [12]; on the other hand, the TMB of LGG is lower than that of GBM [13]. It has also been reported that the correlation of TMB and grade is not significant [14]. The prognostic value and related signaling pathways of TMB in glioma are still not known. In this study, using multi-omics data from TCGA, we systematically analyzed the correlations between TMB and mutational distribution, clinical features and transcriptomic data, revealing potential value for predicting prognosis and the related enrichment pathways of TMB in glioma. With CGGA data, we validated that $T M B$ was an independent biomarker of prognosis. Furthermore, we found that MMR pathways were activated in high-TMB glioma patients without mutations in MMR genes. Finally, we evaluated the correlation between exome sequencing-based TMB and targeted sequencing-based TMB as well as the prognostic value of panel-based TMB, and the results indicated that it was inappropriate to predict TMB and prognosis with pan-cancer targeted sequencing in glioma.

\section{Methods}

\section{Data source}

The training set included the exome sequencing data (level 2, $n=897)$, RNA-seq data $(n=669)$ and clinical data $(n=1105)$ of patients with LGG and GBM from TCGA. Mutational data including variant allele frequencies of mutations were obtained from cBioPortal (http:// www.cbioportal.org) [15, 16]. RNA-seq data were obtained from GlioVis (http://gliovis.bioinfo.cnio.es/) [17]. Clinical data were collected from GlioVis and cBioPortal. The whole-exome sequencing data $(n=286)$, mRNA-seq data $(n=1018)$ and indicated clinical data of the validation set were obtained from CGGA (http://www.cgga. org.cn/index.jsp). Integrated diagnosis was performed according to the World Health Organization (WHO) classification (2016).

\section{TMB (tumor mutational burden)}

The size of the whole-exome genomic region has been defined as $36 \mathrm{Mb}$. The size of the pan-cancer panel genomic region has been defined as $1.06 \mathrm{Mb}$. For the estimation of the TMB of the training set, we used the same approach as was outlined in a recent study [9], i.e., counting all coding somatic base substitutions and indels in the targeted regions, including "stop_/start_lost/ frameshift_/missense_/inframe_" alterations. The software used to estimate TMB was Personal Cancer Genome Reporter software [18]. The TMB of the validation set was calculated with somatic mutations (including single nucleotide variations and short insertions/deletions) identified by SAVI2 as previously described on the CGGA website (http://www.cgga.org.cn/about.jsp).

\section{Statistical analysis}

The Mann-Whitney test was performed to compare the TMBs of two different groups. The Kruskal-Wallis test was used to compare the TMBs of more than two different groups. Spearman's rank correlation test was used to examine the associations between TMB and age and gene expression. The optimal cut-off value was determined by X-tile. Patient survival was analyzed by the Kaplan-Meier method. The following covariates were used in Cox regression analysis (univariate and multivariable): TMB group, sex, WHO grade, histology, IDH status and chromosome $1 \mathrm{p} / 19 \mathrm{q}$ codeletion. $p<0.05$ was considered statistically significant $(" p<0.05, * * p<0.01$, $* * * 0<0.001)$.

\section{GSEA and enrichment map}

GSEA (gene set enrichment analysis) was performed with GSEA software (http://software.broadinstitute.org/ gsea/downloads.jsp) [19], and GO biological process analysis including 4436 gene sets was performed (http:// software.broadinstitute.org/gsea/msigdb/genesets.jsp?coll ection=BP). An enrichment map was used to visualize the results of GSEA according to previously reported methods [20].

\section{Results}

Mutational distribution according to the elevation in TMB

To study the value of TMB in glioma, we first analyzed the types and distributions of nonsynonymous mutations (Supplementary Table 1). As previously reported, the mutation frequencies of $I D H 1$ were higher in LGG than in GBM, and the mutation rates of PTEN and EGFR were higher in GBM than in LGG. We confirmed this conclusion through the analysis of mutational frequencies in LGG and GBM (IDH1, 77\% vs 7\%; PTEN, $5 \%$ vs 29\%; EGFR, 5\% vs 17\%; Supplementary Figs. 1 and 2). Tumors with elevated TMB are enriched for mutations in PTEN or EGFR. Consistently, IDH mutations were primarily found in tumors with low TMB (Fig. 1). We further analyzed the statistical significance of TMB in the mutant and wild-type groups of the 20 genes. With correction for multiple hypotheses, we found that TMB was higher in the PTEN mutant group than in the PTEN 


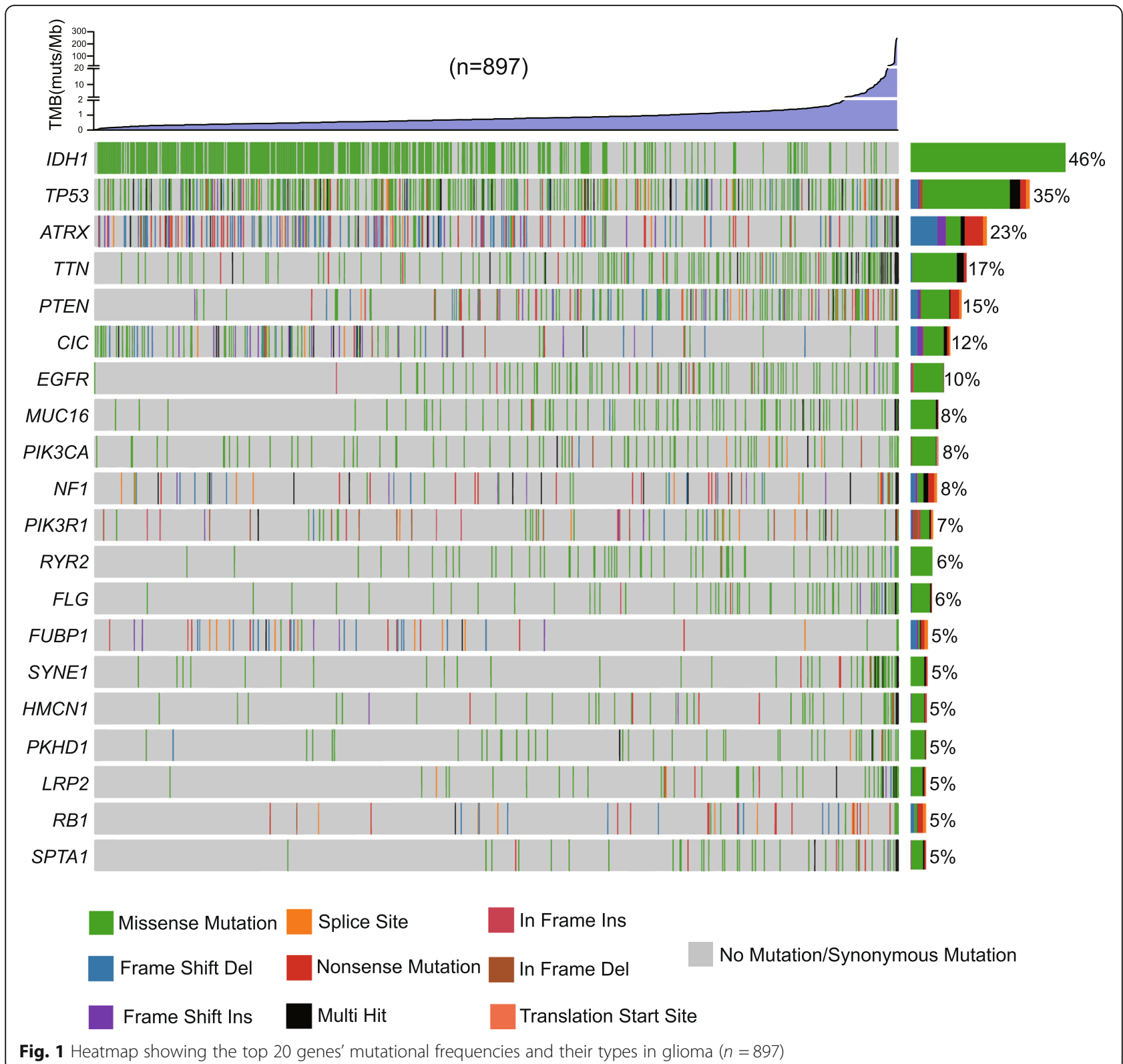

wild-type group but lower in the $I D H 1$ mutant group than in the IDH1 wild-type group (Table 1).

\section{TMB is associated with worse outcomes in glioma} patients

We further analyzed the relationships between TMB and clinical features (Supplementary Table 2, $n=654$ ). Patients without clinical or mutational information were excluded (Fig. 2a). As we expected, TMB increased according to grade (median TMB, 0.47 vs 0.64 vs 0.99 mutations/Mb; Supplementary Fig. 3A). Through ROC analysis, the AUCs for TMB for 2-, 3-, and 5-year survival were $0.775,0.797$, and 0.806 , respectively (Fig. 2b). We determined the cut-off value (between 0.64 and 0.67
mutations/Mb) of TMB with X-tile software, and the patients were divided into $\mathrm{TMB}^{\text {High }}$ and $\mathrm{TMB}^{\mathrm{Low}}$ groups. Overall survival was decreased in patients with a high TMB compared to those with a low TMB (hazard ratio 3.91, 95\% confidence interval 3.33-5.70; $p<0.001$, logrank; Fig. 2c, left). Patients in the TMB ${ }^{\text {High }}$ group exhibited a median overall survival of 23.0 months, whereas those in the $\mathrm{TMB}^{\mathrm{Low}}$ group exhibited a median overall survival of 105.2 months. We confirmed the prognostic effect of TMB with the top $20 \%$ of patients as $\mathrm{TMB}^{\mathrm{High}}$ group and the bottom $80 \%$ of patients as $\mathrm{TMB}^{\mathrm{Low}}$ group (hazard ratio 3.27, 95\% confidence interval 3.93-8.04; $p<0.001$, log-rank; Fig. 2c, right). To determine the prognostic value in the context of established risk 
Table 1 Statistical significance of TMB in mutant and wildtype group of indicated genes in gliomas $(n=897)$

\begin{tabular}{|c|c|c|c|c|c|c|c|}
\hline & Number of mutant group & $\begin{array}{l}\text { Number of } \\
\text { wildtype group }\end{array}$ & $\begin{array}{l}\text { Median TMB of } \\
\text { mutant group }\end{array}$ & $\begin{array}{l}\text { Median TMB of } \\
\text { wildtype group }\end{array}$ & $\begin{array}{l}P \text { value } \\
\text { (Mann-Whitney test) }\end{array}$ & $\begin{array}{l}\text { False discovery rate } \\
\text { (FDR) }\end{array}$ & $\begin{array}{l}\text { Adjust } p \text {-value } \\
\text { (bonferroni) }\end{array}$ \\
\hline IDH1 & 413 & 484 & 0.53 & 0.97 & $2.3 \mathrm{E}-58$ & $4.6 \mathrm{E}-57$ & $4.6 \mathrm{E}-57$ \\
\hline TP53 & 317 & 580 & 0.67 & 0.81 & 5.5E-02 & $5.5 \mathrm{E}-02$ & $1.0 \mathrm{E}+00$ \\
\hline ATRX & 203 & 694 & 0.61 & 0.81 & $1.2 \mathrm{E}-05$ & $1.6 \mathrm{E}-05$ & 2.4E-04 \\
\hline TTN & 149 & 748 & 1.11 & 0.69 & $3.9 \mathrm{E}-20$ & $3.9 \mathrm{E}-19$ & 7.9E-19 \\
\hline PTEN & 136 & 761 & 1.045 & 0.67 & $1.7 \mathrm{E}-18$ & $1.2 \mathrm{E}-17$ & $3.5 \mathrm{E}-17$ \\
\hline $\mathrm{ClC}$ & 106 & 791 & 0.47 & 0.78 & $1.4 \mathrm{E}-11$ & $3.4 \mathrm{E}-11$ & $2.7 \mathrm{E}-10$ \\
\hline EGFR & 89 & 808 & 1.08 & 0.69 & $2.1 \mathrm{E}-14$ & $1.1 \mathrm{E}-13$ & 4.3E-13 \\
\hline MUC16 & 74 & 823 & 1.06 & 0.72 & $5.6 \mathrm{E}-10$ & 1.0E-09 & 1.1E-08 \\
\hline PIK3CA & 74 & 823 & 0.92 & 0.72 & $1.4 \mathrm{E}-03$ & $1.8 \mathrm{E}-03$ & $2.9 \mathrm{E}-02$ \\
\hline NF1 & 70 & 827 & 0.9 & 0.72 & $1.2 \mathrm{E}-02$ & $1.4 \mathrm{E}-02$ & $2.4 \mathrm{E}-01$ \\
\hline PIK3R1 & 60 & 837 & 0.89 & 0.72 & 2.7E-02 & $2.9 \mathrm{E}-02$ & $5.5 \mathrm{E}-01$ \\
\hline RYR2 & 58 & 839 & 0.985 & 0.72 & 2.7E-02 & $2.9 \mathrm{E}-02$ & $5.5 \mathrm{E}-01$ \\
\hline FLG & 57 & 840 & 1.33 & 0.72 & $1.8 \mathrm{E}-13$ & $5.9 \mathrm{E}-13$ & 3.6E-12 \\
\hline FUBP1 & 46 & 851 & 0.5 & 0.75 & $1.8 \mathrm{E}-13$ & $5.9 \mathrm{E}-13$ & 3.6E-12 \\
\hline SYNE1 & 45 & 852 & 4 & 0.72 & $6.8 \mathrm{E}-11$ & 1.5E-10 & 1.4E-09 \\
\hline HMCN1 & 43 & 854 & 1.08 & 0.72 & 2.0E-08 & 3.0E-08 & $3.9 \mathrm{E}-07$ \\
\hline PKHD1 & 42 & 855 & 1.14 & 0.72 & 2.6E-07 & 3.7E-07 & $5.2 \mathrm{E}-06$ \\
\hline LRP2 & 41 & 856 & 1.25 & 0.72 & $1.2 \mathrm{E}-10$ & $2.4 \mathrm{E}-10$ & 2.4E-09 \\
\hline RB1 & 41 & 856 & 1.22 & 0.72 & $9.9 \mathrm{E}-09$ & 1.7E-08 & $2.0 \mathrm{E}-07$ \\
\hline SPTA1 & 41 & 856 & 1.25 & 0.72 & $3.0 \mathrm{E}-12$ & 8.7E-12 & $6.1 \mathrm{E}-11$ \\
\hline
\end{tabular}

factors, we performed survival analysis in different subgroups (Fig. 2d-g). TMB was significantly associated with poor outcomes in the indicated subgroups except for the glioblastoma, WHO grade IV and IDH wildtype subgroups. To test whether TMB was an independent biomarker for prognosis, we performed Cox regression analysis in an independent validation set. In the univariate analysis, age, grade, histology, IDH status, chromosome $1 \mathrm{p} / 19 \mathrm{q}$ codeletion and TMB were statistically significantly associated with overall survival $(p<0.01)$ (Table 1, Supplementary Table 3). In the multivariable analysis, grade, chromosome $1 \mathrm{p} / 19 \mathrm{q}$ codeletion and
TMB were independently associated with overall survival (Table 2). Furthermore, we analyzed the distribution of clinical features accompanied by an elevated TMB (Fig. $2 \mathrm{~g})$. TMB was significantly increased in older patients but was not associated with sex (Supplementary Fig. 3B, C). In the subgroup analysis of integrated diagnosis, TMB was found to be elevated in the anaplastic astrocytoma $I D H$ wild-type group compared to the other astrocytoma group (Supplementary Fig. 3D). TMB was also increased for the classic-like and mesenchymal-like subtypes compared to other $I D H$ wild-type subtypes and for the G-CIMP-low subtype compared to other $I D H$

Table 2 Univariate and multivariable Cox regression analyses of factors associated with overall survival in glioma patients

\begin{tabular}{|c|c|c|c|c|}
\hline \multirow[t]{2}{*}{ Variable } & \multicolumn{2}{|l|}{ Univariate analysis } & \multicolumn{2}{|c|}{ Multivariable analysis } \\
\hline & $\mathrm{HR}(95 \% \mathrm{Cl})$ & $P^{*}$ & HR $(95 \%$ Cl) & $\mathrm{P}$ \\
\hline Age & $1.86(1.19$ to 2.90$)$ & $<0.01$ & I & I \\
\hline Gender & $1.31(0.87$ to 1.98$)$ & 0.19 & / & / \\
\hline Grade & $2.61(1.95$ to 3.49$)$ & $<0.01$ & $2.08(1.53$ to 2.82$)$ & $<0.01$ \\
\hline Histology & $1.45(1.12$ to 1.86$)$ & $<0.01$ & / & / \\
\hline IDH.status & $0.36(0.24$ to 0.54$)$ & $<0.01$ & / & / \\
\hline Chr.1p/19q.codeletion & $0.12(0.05$ to 0.27$)$ & $<0.01$ & $0.15(0.06$ to 0.36$)$ & $<0.01$ \\
\hline TMB group & $2.58(1.67$ to 3.97$)$ & $<0.01$ & $1.90(1.21$ to 2.98$)$ & $<0.01$ \\
\hline
\end{tabular}

"All statistical tests were two-sided. $C l$ confidence interval, $H R$ hazard ratio, $T M B$ cut-off value $=0.655$ mutations $/ \mathrm{Mb}$ 

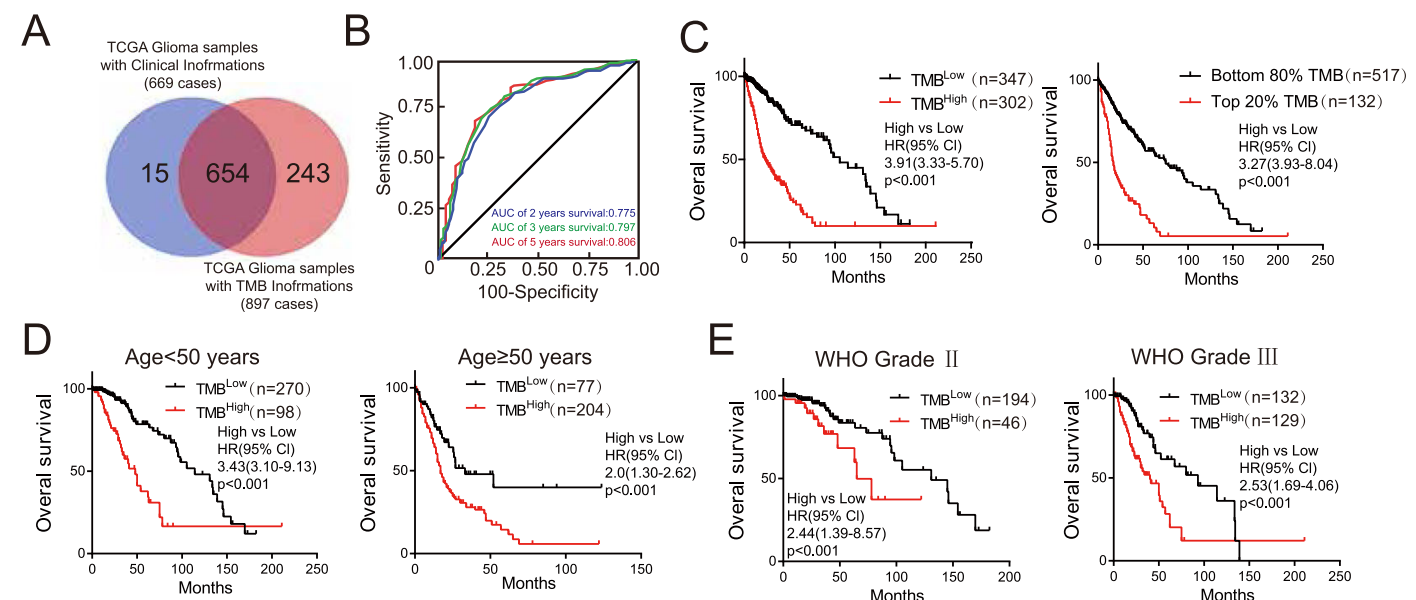

E
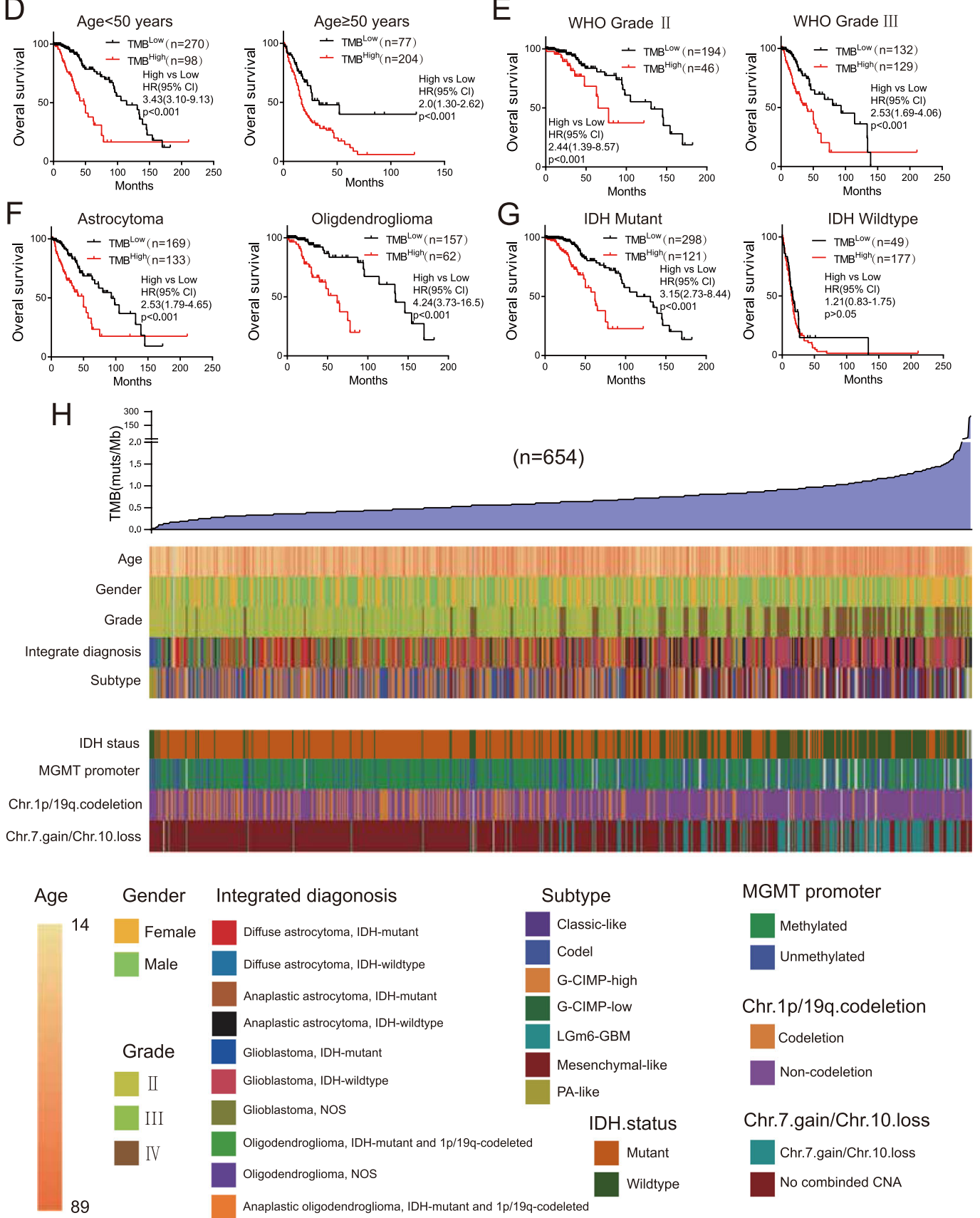

Integrated diagonosis

Subtype

Classic-like

Codel

G-CIMP-high

G-CIMP-low

LGm6-GBM

Mesenchymal-like

PA-like

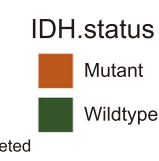

Anaplastic oligodendroglioma, IDH-mutant and 1p/19q-codeleted

Anaplastic oligodendroglioma, NOS
MGMT promoter

Methylated

Unmethylated

Chr.1p/19q.codeletion

Codeletion

Non-codeletion

Chr.7.gain/Chr.10.loss

Chr.7.gain/Chr.10.loss

No combinded CNA

Fig. 2 (See legend on next page.) 
(See figure on previous page.)

Fig. $2 \mathrm{TMB}$ is associated with worse outcomes in glioma patients. a Venn diagram of the patients included in further analysis. $\mathbf{b}$ ROC analysis of 2-, 3-, and 5-year survival according to TMB. c Kaplan-Meier curves of the overall survival of glioma patients $(n=649,5$ patients lacked survival information) with high TMB (TMB ${ }^{\text {High }}$ ) versus those with low TMB (TMB $\left.{ }^{\text {Low }}\right)$. The cut-off value of the left panel was between 0.64 and 0.67 . The right panel had the highest $20 \%$. $\mathbf{d}$-g Survival analysis was performed in the indicated subgroups. $\mathbf{h}$ Heatmap showing the distribution of clinical features and genetic characteristics of the glioma specimens $(n=654)$

mutant subtypes (Supplementary Fig. 3E) [5]. Mutational analysis revealed that the patients exhibiting an unmethylated MGMT promoter, non-codeletion of $1 \mathrm{p} /$ $19 \mathrm{q}$ and Chr.7.gain/Chr.10.loss exhibited a higher TMB (Supplementary Fig. 3F). Overall, these data indicated that TMB could be an independent prognostic biomarker of glioma.

\section{$\mathrm{TMB}^{\mathrm{High}}$ gliomas exhibit elevated proliferative activity and immune responses}

To clarify the mechanism of the association between the TMB and poor outcomes of glioma patients, we analyzed the data from patients with TMB information and RNA-seq data $(n=654)$. Gene set enrichment analysis (GSEA) coupled with enrichment map analysis was performed to visualize the enriched biological processes. The $\mathrm{TMB}^{\mathrm{High}}$ group was enriched in transcriptional programs related to the cell cycle, DNA replication and immune effector processes. In contrast, the transcriptional programs of adenylate cyclase activity and synaptic transmission were enriched in the $\mathrm{TMB}^{\mathrm{Low}}$ group (Fig. 3a, b). These transcriptomics data indicated that high-TMB gliomas exhibit intensive proliferative activity, which might result in a worse prognosis. We performed GSEA in different subgroups and found that the $\mathrm{TMB}^{\text {High }}$ group was enriched in transcriptional programs related to the cell cycle when we controlled for age, WHO grade, histology and IDH status (Supplementary Fig. 4). GSEA of the validation set also confirmed the results (Fig. 3c). Furthermore, TMB exhibited a modest correlation with the inflammatory biomarkers of checkpoint inhibitor-based immunotherapy (Fig. 3d), which was consistent with the findings of previous reports based on the pan-cancer dataset [6].

\section{High TMB is associated with the mismatch repair pathway} in gliomas without mutations in classical MMR genes

It has been reported that MMR (mismatch repair) deficiency is associated with a higher TMB in gliomas [14], and we confirmed this finding in the TCGA dataset. Only $3.6 \%$ of glioma patients harbored MMR gene mutations (32 of 897 glioma patients). TMB was elevated in patients exhibiting $M L H 1, M S H 2, M S H 6$, PMS2, POLD1 or POLE gene mutations (Fig. 4a). We further performed GSEA in patients without mutations in MMR genes to confirm whether high TMB was associated with low mismatch repair function. Interestingly, mismatch repair-associated transcriptional programs were also enriched in the $\mathrm{TMB}^{\text {High }}$ group but not in the $\mathrm{TMB}^{\text {Low }}$ group (Fig. 4b, c). The correlation analysis of TMB and the expression of MMR genes further demonstrated that a high TMB was associated with the expression of MLH1, MSH2, MSH6, POLD1 and POLE in gliomas without MMR mutations (Fig. 4d). These data indicated that when the MMR genes are not mutated, TMB exhibits a positive correlation with MMR function.

\section{Pan-cancer targeted sequencing cannot predict prognosis in glioma}

Considering the cost of exome sequencing, targeted sequencing is widely used to predict TMB in pan-cancer analyses. We calculated TMB using 468 genes (Supplementary Table 4) from MSK-IMPACT [11] and analyzed the Spearman correlation with TMB calculated on the basis of exome sequencing (Fig. 5a, Supplementary Table 5). Interestingly, unlike other cancer types, the correlation between panel-based TMB and exome sequencing-based TMB was moderate in glioma $(r=0.3105)$. This result was confirmed with two other panels (Supplementary Table 4) that are used in China (Supplementary Fig. 5, $r=0.2753 / 0.3461$ ). We further performed ROC analysis for panel-based TMB (Fig. 5b), and the result was significantly different from the ROC of exome sequencing-based TMB (Fig. 2b). We performed survival analysis with different TMB cut-off values. Only when the cut-off value was between 0.64 and 0.67 was TMB negatively associated with overall survival (Fig. 5c), which was not consistent with previous results (Fig. 2c). With the other cut-off value, TMB was not correlated with overall survival (Fig. 5c). These data indicated that, at least in glioma, pan-cancer panel-based TMB cannot represent exome sequencing-based TMB and is not suitable for prognosis.

\section{Discussion}

In this study, to understand the value of TMB in glioma, we first analyzed the relationship between TMB and the mutation distribution. For most genes, TMB was elevated in the individual gene mutant group compared to the individual gene wild-type group. For example, TMB was higher in the PTEN mutant group than in the PTEN wild-type group. It is reasonable as TMB is an aggregate of mutations that result in protein alterations. However, for genes that were mutated in lower-grade glioma, such as $I D H 1$, TMB was lower in the IDH1 mutant group 


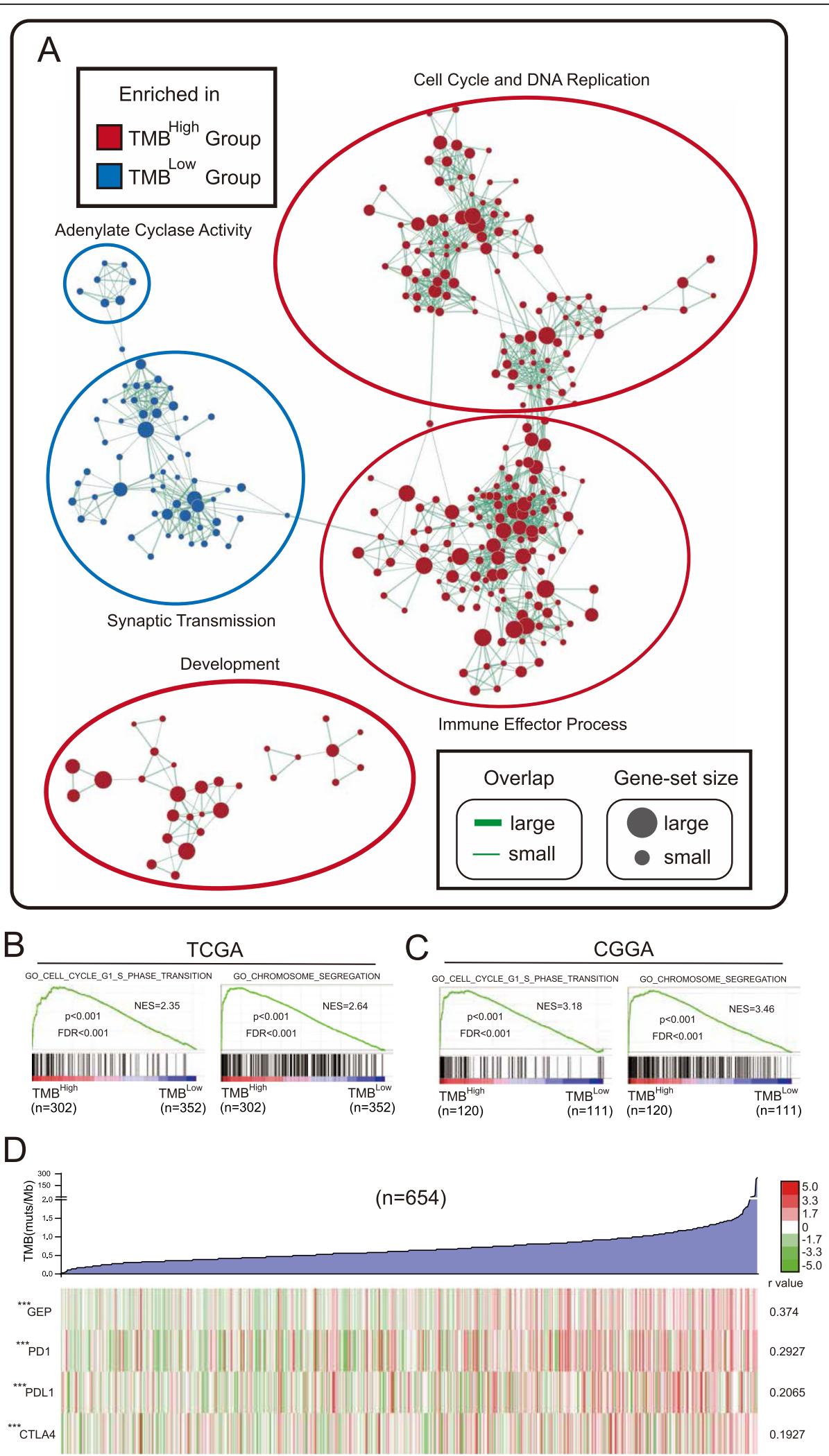

Fig. 3 (See legend on next page.) 
(See figure on previous page.)

Fig. $3 \mathrm{TMB}^{\text {High }}$ gliomas exhibit increased proliferative activity and immune responses. a GO biological progress enriched by GSEA in the TMB ${ }^{\text {High }}$ group ( $n=302)$ versus the TMB ${ }^{\text {Low }}$ group $(n=352)$ using an enrichment map. Node size represents the number of genes in the gene sets. Line width represents the number of overlapping genes. b Representative GSEA enrichment plots in (a). The NES (normalized enrichment score), $p$ value and FDR (false discovery rate) were calculated with GSEA software. c GSEA of the validation set (CGGA) in the indicated gene sets. $\mathbf{d}$ The heatmap showing the distribution and correlation of the indicated gene set/genes in glioma specimens was visualized using Java Tree-view. Spearman's $r$ value and significance were calculated

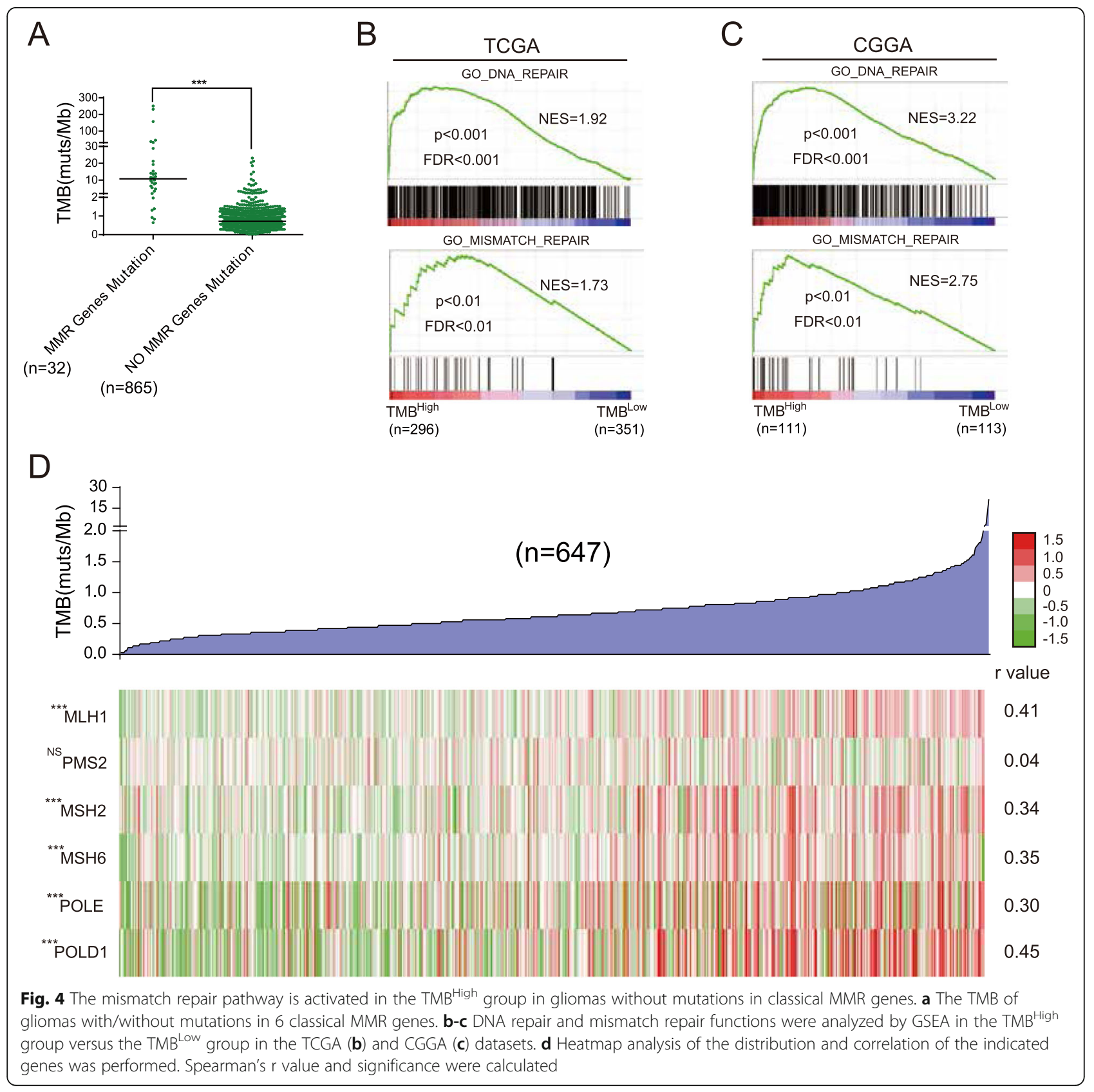


A

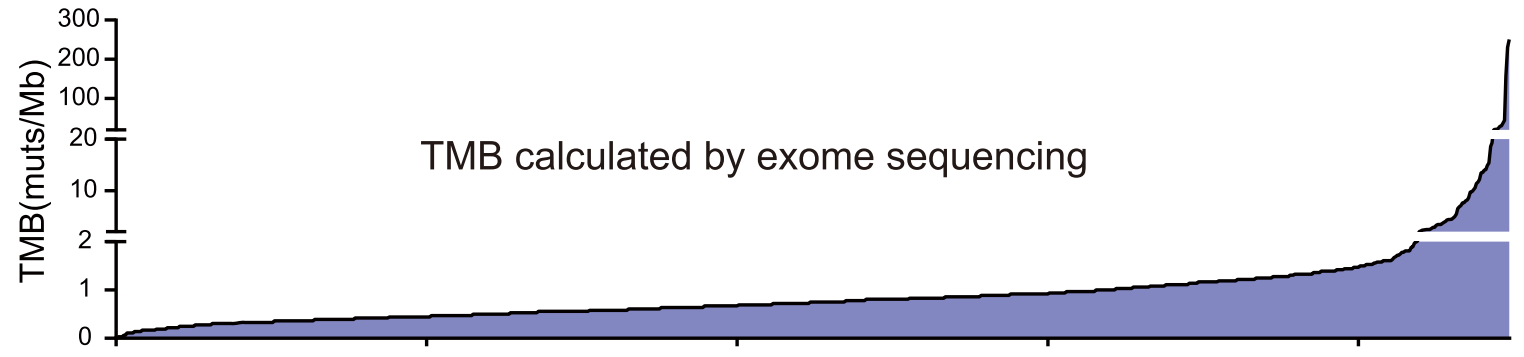

Spearman $r=0.3105 p<0.001(n=897)$
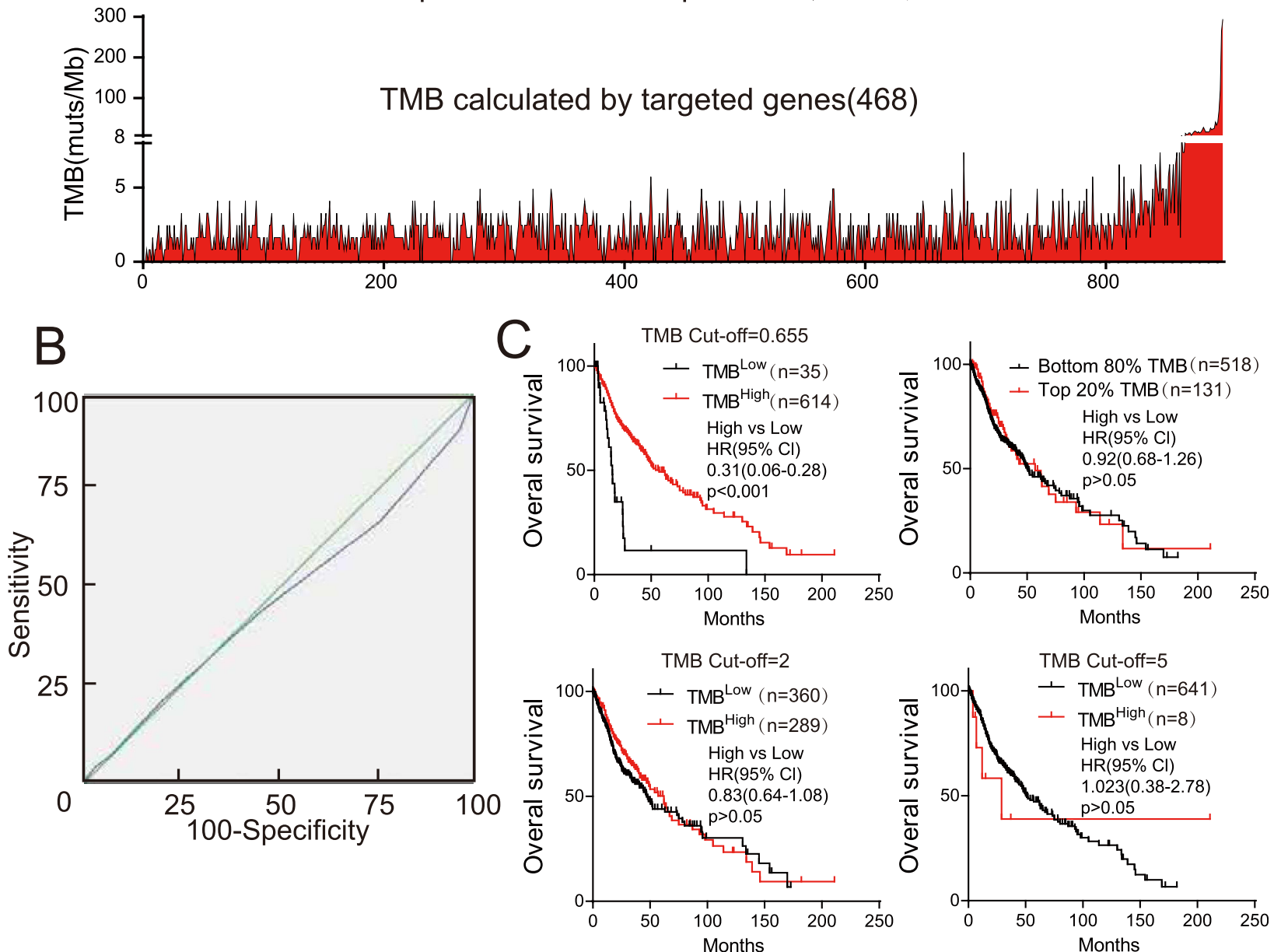

Fig. 5 Pan-cancer targeted sequencing-based TMB cannot predict prognosis in glioma. a Correlation of TMB calculated on the basis of exome sequencing and targeted genes. The list of genes is from the FDA-approved targeted next generation sequencing panel (MSK-IMPACT). Spearman's $r$ value and significance were calculated. b AUC analysis with TMB calculated using the MSK-IMPACT panel. c Survival analysis was performed with the indicated cut-off value

than in the $I D H 1$ wild-type group. Through the analysis of clinicopathological parameters, we found that TMB was significantly elevated with an increasing grade and was associated with the poor survival of patients. These results were validated with the CGGA dataset through multivariable Cox regression analysis. According to enrichment map analysis with GSEA, the $\mathrm{TMB}^{\mathrm{High}}$ group exhibited activation of cell proliferation. As reported in pan-cancer studies, the correlation between $\mathrm{TMB}$ and $\mathrm{T}$ cell immunity was moderate, and TMB was elevated in the MMR gene mutant group. Interestingly, in the MMR gene wild-type group, the transcriptional 
programs of MMR enriched in the $\mathrm{TMB}^{\mathrm{High}}$ group and TMB were positively correlated with the expression of MMR genes. Finally, by analyzing pan-cancer targeted sequencing, it was found that TMB based on panel analysis was not highly correlated with TMB calculated by exome sequencing in glioma. No prognostic value existed for targeted sequencing-based TMB.

The limitations of this study include optimal cut-off value and experimental validation. The conclusion could be totally different with different cut-off values. To address this concern, we analyzed the relative risk with different cut-off values by X-tile software. The optimal cut-off value was between 0.64 and 0.67 , and we further tested the prognostic value with an independent dataset by multivariable analysis. GSEA is a promising method to explore the relationship between risk factors and signaling pathways with expression profiling data; however, causal links still need experimental validation. Although we confirmed the activation of cell proliferation in the $\mathrm{TMB}^{\text {High }}$ group with the CGGA dataset, it is essential to test this conclusion with primary glioma cells in vitro and in vivo.

With the development of large-scale sequencing, thousands of somatic mutations have been revealed in cancer samples. Different cancer types exhibit distinct mutational signatures [12]. The mutational landscape is reported to illustrate driver mutations and can be used to develop individualized treatments [21]. In glioma, the reported TMB is paradoxical. The mutational load calculated by exome sequencing is associated with the tumor grade and age of patients [22]. However, in another cohort, targeted sequencing-based TMB was not correlated with grade [14]. To study the application of targeted sequencing in the prediction of TMB in glioma, we analyzed the correlation between exome sequencing-based TMB and targeted sequencing-based TMB in a TCGA cohort. Although the correlation was significant, the coefficient was modest. For most gliomas with a TMB $<2$, the targeted sequencing-based calculation could not exactly predict TMB. Although targeted sequencing is more economical than exome sequencing, pan-cancer targeted sequencing-based TMB may be inappropriate for predicting the prognosis of glioma patients. Gliomacustomized panels should be designed to precisely predict the mutational load.

Emerging data imply that neoantigens resulting from nonsynonymous mutations could serve as potential biomarkers for checkpoint blockade therapy. The mutation that results in tumor initiation could also be targeted by the immune system [15]. In a mouse model, radiation plus anti-PD-1 treatments improved survival compared to radiation alone [23]. With immune checkpoint inhibitor treatment, the tumor size of glioblastomas with hypermutation was significantly reduced [24]. However, the failure of CheckMate-143 indicates that nivolumab does not improve the OS of patients with recurrent glioblastoma compared to bevacizumab treatment [25]. These data imply that the mutational state should be analyzed before immune checkpoint inhibitor treatment. We analyzed the relationship between TMB and other biomarkers of immune checkpoint inhibitors and illustrated the possible mechanism by which TMB is associated with poor survival. Our work revealed potential biomarkers for improving survival in glioma patients. However, our data were mainly obtained from patients treated with routine chemoradiotherapy. TMB should be tested in patients treated with immune checkpoint inhibitors.

\section{Conclusions}

1. TMB is associated with shorter overall survival in glioma patients.

2. Proliferative activity and the immune response are activated in $\mathrm{TMB}^{\text {High }}$ gliomas.

3. TMB was higher in the MMR gene mutant group, but the MMR pathway was enriched in the $\mathrm{TMB}^{\mathrm{High}}$ group.

4. Pan-cancer targeted sequencing-based TMB cannot predict prognosis in glioma.

\section{Supplementary information}

Supplementary information accompanies this paper at https://doi.org/10 1186/s12885-020-6658-1.

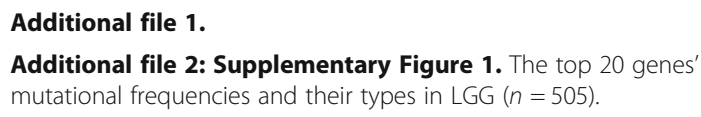

Additional file 3: Supplementary Figure 2. The top 20 genes' mutational frequencies and their types in GBM $(n=392)$.

\section{Additional file 4.}

Additional file 5: Supplementary Figure 3. TMB was associated with different grades $(A)$, ages $(B)$, sexes $(C)$, integrated diagnoses $(D)$, subtypes (E) and mutational statuses (F) in glioma. Statistical significance was calculated with the Mann-Whitney test for two groups and with the Kruskal-Wallis test for more than two groups.

\section{Additional file 6.}

Additional file 7: Supplementary Figure 4. The transcriptional programs of the cell cycle were enriched in the $T M B^{\text {Low }}$ group at different ages (A), WHO grades (B), histology (C) and IDH statuses (D). The NES (normalized enrichment score), $p$ value and FDR (false discovery rate) were calculated with GSEA software.

Additional file $\mathbf{8}$.

Additional file 9.

Additional file 10: Supplementary Figure 5. The correlation of TMB calculated on the basis of exome sequencing and targeted genes. Gene list from two local panels used in pan-cancer analysis. Spearman's $r$ value and significance were calculated.

\section{Abbreviations}

BP: Biological process; CGGA: Chinese Glioma Genome Atlas; GBMs: Glioblastomas; GEPs: Gene expression profiles; GO: Gene Ontology; GSEA: Gene set enrichment analysis; IDH: Isocitrate dehydrogenase; 
LGGs: Lower-grade gliomas; MLH1: MutL Homolog 1; MMR: Mismatch repair; MSH2: MutS Homolog 2; MSH6: MutS Homolog 6; MSK-IMPACT: Memorial Sloan-Kettering Cancer Center-Integrated Mutation Profiling of Actionable Cancer Targets; OS: Overall survival; PMS2: PMS1 Homolog 2, Mismatch Repair System Component; POLD1: DNA Polymerase Delta 1, Catalytic Subunit; POLE: DNA Polymerase Epsilon, Catalytic Subunit; PTEN: Phosphate and tension homology deleted on chromosome ten; ROC: Receiver operating characteristic; TCGA: The Cancer Genome Atlas; TMB: Tumor mutational burden; WHO: World Health Organization

\section{Acknowledgments}

The authors gratefully acknowledge Dr. Zhengqing Lei from Zhongda Hospital of Southeast University for the support of this research by providing statistical advice.

\section{Authors' contributions}

TL conceived this work, performed the statistical analysis, and wrote the paper. LW collected and preprocessed the data from TCGA. LW generated the panel for subtype analyses and panel-based TMB. YYT and WW collected and preprocessed the data from CGGA. YX performed GSEA with the CGGA data. First $Y L$, second $Y L, J G, Y H T, M L$ and SD helped to interpret the results. $T L, X Z$ and $Y S$ revised the manuscript. TL supervised the whole study. All authors have read and approved the final manuscript.

\section{Funding}

This research was supported by grants from the National Natural Science Foundation of China $(81602196,81874087)$, the National Key R\&D Program of China (2016YFA0101200, 2016YFC1201801) and the Chongqing Basic Research Project (cstc2016jcyjA2194). The funds mentioned were used for the design of the study, the collection, analysis, and interpretation of the data as well as the writing of the manuscript.

\section{Availability of data and materials}

The datasets generated and/or analyzed during the current study are available in the TCGA (http://www.cbioportal.org), GlioVis (http://gliovis. bioinfo.cnio.es/) and CGGA (http://gliovis.bioinfo.cnio.es/) databases.

\section{Ethics approval and consent to participate}

Not applicable.

\section{Consent for publication}

Not applicable.

\section{Competing interests}

The authors declare that they have no competing interests.

\section{Author details}

${ }^{1}$ Institute of Pathology and Southwest Cancer Center, Southwest Hospital, Third Military Medical University (Army Medical University) and Key Laboratory of Tumor Immunopathology, Ministry of Education of China, Chongqing 400038, China. ${ }^{2}$ Bio-Bank of Southwest Hospital, Third Military Medical University (Army Medical University), Chongqing 400038, China.

Received: 15 July 2019 Accepted: 20 February 2020

Published online: 12 March 2020

\section{References}

1. Cancer Genome Atlas Research N, Brat DJ, Verhaak RG, Aldape KD, Yung WK, Salama SR, Cooper LA, Rheinbay E, Miller CR, Vitucci M, et al. Comprehensive, Integrative Genomic Analysis of Diffuse Lower-Grade Gliomas. N Engl J Med. 2015;372:2481-98. https://doi.org/10.1056/ NEJMoa1402121.

2. Ceccarelli M, Barthel FP, Malta TM, Sabedot TS, Salama SR, Murray BA, Morozova O, Newton Y, Radenbaugh A, Pagnotta SM, et al. Molecular profiling reveals biologically discrete subsets and pathways of progression in diffuse Glioma. Cell. 2016;164:550-63. https://doi.org/10.1016/j.cell.2015.12.028.

3. Eckel-Passow JE, Lachance DH, Molinaro AM, Walsh KM, Decker PA, Sicotte H, Pekmezci M, Rice T, Kosel ML, Smirnov IV, et al. Glioma groups based on 1p/19q, IDH, and TERT promoter mutations in tumors. N Engl J Med. 2015; 372:2499-508. https://doi.org/10.1056/NEJMoa1407279.
4. Louis DN, Perry A, Reifenberger $G$, von Deimling A, Figarella-Branger D, Cavenee WK, Ohgaki H, Wiestler OD, Kleihues P, Ellison DW. The 2016 World Health Organization classification of tumors of the central nervous system: a summary. Acta Neuropathol. 2016;131:803-20. https://doi.org/10.1007/ s00401-016-1545-1.

5. Samstein $R M$, Lee $C H$, Shoushtari $A N$, Hellmann MD, Shen $R$, Janjigian $Y Y$, Barron DA, Zehir A, Jordan EJ, Omuro A, et al. Tumor mutational load predicts survival after immunotherapy across multiple cancer types. Nat Genet. 2019;51:202-6. https://doi.org/10.1038/s41588-018-0312-8.

6. Cristescu R, Mogg R, Ayers M, Albright A, Murphy E, Yearley J, Sher X, Liu $X Q$, Lu H, Nebozhyn M, et al. Pan-tumor genomic biomarkers for PD-1 checkpoint blockade-based immunotherapy. Science. 2018;362. https://doi. org/10.1126/science.aar3593.

7. Devarakonda S, Rotolo F, Tsao MS, Lanc I, Brambilla E, Masood A, Olaussen KA, Fulton R, Sakashita S, McLeer-Florin A, et al. Tumor mutation burden as a biomarker in resected non-small-cell lung Cancer. J Clin Oncol. 2018;36: 2995-3006. https://doi.org/10.1200/JCO.2018.78.1963.

8. Hwang WL, Wolfson RL, Niemierko A, Marcus KJ, DuBois SG, Haas-Kogan D. Clinical impact of tumor mutational burden in neuroblastoma. J Natl Cancer Inst. 2018. https://doi.org/10.1093/jnci/djy157.

9. Chalmers ZR, Connelly CF, Fabrizio D, Gay L, Ali SM, Ennis R, Schrock A, Campbell B, Shlien A, Chmielecki J, et al. Analysis of 100,000 human cancer genomes reveals the landscape of tumor mutational burden. Genome Med. 2017;9:34. https://doi.org/10.1186/s13073-017-0424-2.

10. Johanns TM, Miller CA, Dorward IG, Tsien C, Chang E, Perry A, Uppaluri R, Ferguson C, Schmidt RE, Dahiya S, et al. Immunogenomics of Hypermutated Glioblastoma: a patient with Germline POLE deficiency treated with checkpoint blockade immunotherapy. Cancer Discov. 2016;6:1230-6. https://doi.org/10.1158/2159-8290.CD-16-0575.

11. Rizvi H, Sanchez-Vega F, La K, Chatila W, Jonsson P, Halpenny D, Plodkowski A, Long N, Sauter JL, Rekhtman N, et al. Molecular determinants of response to anti-programmed cell death (PD)-1 and anti-programmed death-ligand 1 (PD-L1) blockade in patients with non-small-cell lung Cancer profiled with targeted next-generation sequencing. J Clin Oncol. 2018;36:633-41. https://doi.org/10.1200/JCO.2017.75.3384.

12. Alexandrov LB, Nik-Zainal S, Wedge DC, Aparicio SA, Behjati S, Biankin AV, Bignell GR, Bolli N, Borg A, Borresen-Dale AL, et al. Signatures of mutational processes in human cancer. Nature. 2013;500:415-21. https://doi.org/10. 1038/nature12477.

13. Schumacher TN, Schreiber RD. Neoantigens in cancer immunotherapy. Science. 2015;348:69-74. https://doi.org/10.1126/science.aaa4971.

14. Hodges TR, Ott M, Xiu J, Gatalica Z, Swensen J, Zhou S, Huse JT, de Groot J, Li S, Overwijk WW, et al. Mutational burden, immune checkpoint expression, and mismatch repair in glioma: implications for immune checkpoint immunotherapy. Neuro-Oncology. 2017;19:1047-57. https://doi.org/10.1093/ neuonc/nox026

15. Cerami E, Gao J, Dogrusoz U, Gross BE, Sumer SO, Aksoy BA, Jacobsen A, Byrne CJ, Heuer ML, Larsson E, et al. The cBio cancer genomics portal: an open platform for exploring multidimensional cancer genomics data. Cancer Discov. 2012;2:401-4. https://doi.org/10.1158/2159-8290.CD-12-0095.

16. Gao J, Aksoy BA, Dogrusoz U, Dresdner G, Gross B, Sumer SO, Sun Y,

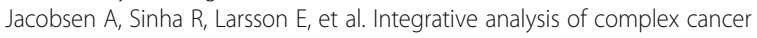
genomics and clinical profiles using the cBioPortal. Sci Signal. 2013;6:pl1. https://doi.org/10.1126/scisignal.2004088.

17. Bowman RL, Wang Q, Carro A, Verhaak RG, Squatrito M. GlioVis data portal for visualization and analysis of brain tumor expression datasets. NeuroOncology. 2017;19:139-41. https://doi.org/10.1093/neuonc/now247.

18. Nakken S, Fournous G, Vodak D, Aasheim LB, Myklebost O, Hovig E. Personal Cancer genome reporter: variant interpretation report for precision oncology. Bioinformatics. 2018;34:1778-80. https://doi.org/10.1093/ bioinformatics/btx817.

19. Subramanian A, Tamayo P, Mootha VK, Mukherjee S, Ebert BL, Gillette MA, Paulovich A, Pomeroy SL, Golub TR, Lander ES, et al. Gene set enrichment analysis: a knowledge-based approach for interpreting genome-wide expression profiles. Proc Natl Acad Sci U S A. 2005;102:15545-50. https://doi.org/10.1073/pnas.0506580102.

20. Merico D, Isserlin R, Stueker O, Emili A, Bader GD. Enrichment map: a networkbased method for gene-set enrichment visualization and interpretation. PLoS One. 2010;5:e13984. https://doi.org/10.1371/journal.pone.0013984.

21. Kandoth C, McLellan MD, Vandin F, Ye K, Niu B, Lu C, Xie M, Zhang Q, McMichael JF, Wyczalkowski MA, et al. Mutational landscape and 
significance across 12 major cancer types. Nature. 2013;502:333-9. https://doi.org/10.1038/nature12634.

22. Draaisma K, Wijnenga MM, Weenink B, Gao Y, Smid M, Robe P, van den Bent MJ, French PJ. Pl3 kinase mutations and mutational load as poor prognostic markers in diffuse glioma patients. Acta Neuropathol Commun. 2015;3:88. https://doi.org/10.1186/s40478-015-0265-4.

23. Zeng J, See AP, Phallen J, Jackson CM, Belcaid Z, Ruzevick J, Durham N, Meyer C, Harris TJ, Albesiano E, et al. Anti-PD-1 blockade and stereotactic radiation produce long-term survival in mice with intracranial gliomas. Int J Radiat Oncol Biol Phys. 2013;86:343-9. https://doi.org/10.1016/j.jijobp.2012. 12.025.

24. Bouffet E, Larouche V, Campbell BB, Merico D, de Borja R, Aronson M, Durno C, Krueger J, Cabric V, Ramaswamy V, et al. Immune checkpoint inhibition for Hypermutant Glioblastoma Multiforme resulting from Germline Biallelic mismatch repair deficiency. J Clin Oncol. 2016;34:2206-11. https://doi.org/ 10.1200/JCO.2016.66.6552.

25. Filley AC, Henriquez M, Dey M. Recurrent glioma clinical trial, CheckMate143: the game is not over yet. Oncotarget. 2017;8:91779-94. https://doi.org/ 10.18632/oncotarget.21586.

\section{Publisher's Note}

Springer Nature remains neutral with regard to jurisdictional claims in published maps and institutional affiliations.

Ready to submit your research? Choose BMC and benefit from:

- fast, convenient online submission

- thorough peer review by experienced researchers in your field

- rapid publication on acceptance

- support for research data, including large and complex data types

- gold Open Access which fosters wider collaboration and increased citations

- maximum visibility for your research: over $100 \mathrm{M}$ website views per year

At $\mathrm{BMC}$, research is always in progress.

Learn more biomedcentral.com/submissions 